\title{
An efficient synthesis of new benzohydrazide and 1,3-thiazine fused s-triazines as potential antimicrobial agents
}

\author{
G. M. Malik ${ }^{1, *}$, T. V. Patel ${ }^{2}$ \\ ${ }^{1}$ Associate Professor, ${ }^{2} \mathrm{PhD}$ Student, Dept. of Chemistry, Navyug Science College, Surat, Gujarat, India \\ *Corresponding Author: \\ Email: talha.pate19@gmail.com
}

\begin{abstract}
As a part of our endeavor toward the synthesis of new heterocyclic bioactive agents, some new substituted 1,3,5 triazine derivatives with 4-nitrobenzohydrazide and 6-(4-methoxyphenyl)-4-phenyl-6H-1,3-thiazin-2-amine and substituted thiourea were reacted and evaluated for their in vitro antimicrobial activity against Gram positive and Gram negative strains using a micro dilution procedure. Synthesized compounds T1GE to T15GE showed to be effective with MIC ( $\mu \mathrm{g} / \mathrm{mL}$ ), among them T5GE, T8GE, T9GE and T14GE showed excellent activity against a panel of microorganisms. The newly synthesized compounds were characterized using IR, ${ }^{1} \mathrm{H}-\mathrm{NMR},{ }^{13} \mathrm{C}-\mathrm{NMR}$, MASS Analysis.
\end{abstract}

Keywords: Cyanuric Chloride, 4-nitrobenzohydrazide, 6-(4-methoxyphenyl)-4-phenyl-6H-1, 3-thiazin-2-amine, Different thiourea derivatives and antimicrobial activity.

\section{Introduction}

After years of misuse and overuse of antibiotics, bacteria are becoming antibiotic resistant therefore recent efforts have been directed toward exploring novel antibacterial agents ${ }^{1}$. Since last two decades there are many antibiotics and chemotherapeutics available. The challenging therapeutic problems of the treatment of infectious diseases were still remains due to the inexorable increase and spread of multidrug-resistant strains. So as to diminish the speedy multidrugresistance in pathogenic microbes, there is an appearance necessitate for the development of new module of antimicrobial agents. ${ }^{2}$ For the development of drug resistance, new agents should rather consist of chemical characteristics that clearly differ from those of presented agents. Nowadays the new finding and commercial development of several therapeutic agents ${ }^{3}$ afford consistently effective treatment for many infectious diseases which had previously caused widespread humanity and morbidity. In recent year in organic synthesis, the chemistry of substituted benzohydrazide derivatives are one of the important subjects. The major problem in the helpful antibacterial and antifungal treatment is increasing conflict of microorganisms to currently available antimicrobial drugs. ${ }^{4-7}$ Benzohydrazide have been reported to possess various biological activities such as antileishmanial, ${ }^{8}$ anti-inflammatory, ${ }^{9}$ anticancer, ${ }^{10}$ antimycobacterial, ${ }^{11}$ anti-tumoral studies was reviewed by Rollas et $a .^{12}$ Thiazine derivatives are an important class of heterocyclic compounds reported to possess a wide spectrum of biological properties such as anticonvulsant, ${ }^{13}$ anti-inflammatory, ${ }^{14-16}$ anticancer, ${ }^{17}$ antidiabetic, ${ }^{18}$ analgesic, ${ }^{19}$ immunotropic. ${ }^{20}$ It has been reported that, s-triazine ring skeleton possesses a broad spectrum of biological and pharmaceutical activities, such as antimicrobial, antifungal, antibacterial, anticancer, anti HIV, anti-inflammatory, antituberculosis, antimalarial etc. ${ }^{21-29}$ Cyanuric chloride and various amines with good antibacterial properties were used. Therefore, it is predicted that chemical entities with 4-nitrobenzohydrazide, 6-(4-methoxyphenyl)-4phenyl-6H-1, 3-thiazin-2-amine, different thiourea and s-triazine moieties would result in compounds of interesting biological activities. In view of these findings, we have attempted to incorporate all these four biologically active components together to give a confined structure as describe below in reaction scheme. All synthesized compounds for evaluating their antibacterial and antifungal activities.

Previously, we were also reported synthesis, characterization and antimicrobial evaluation of 4-((4((5-benzyl-1,3,4-thiadiazol-2-yl)amino)-6(phenylamino)1,3,5-triazin-2-yl)amino)-6-(tert-butyl)3 (methylthio)-1,2,4-triazin-5(4H)-one derivatives ${ }^{30}$, 1 (4-((5-methyl-1,3,4-thiadiazol-2-yl-)amino)-6-((4phenylthiazol-2-yl)amino-1,3,5-triazine-yl)-3phenylurea $^{31}$, 4-((4-((5-benzyl-1,3,4-thiadiazol-2yl)amino)-6-(phenyl amino) 1,3,5-triazin-2-yl)amino)6-(tert-butyl)-3-(methylthio)-1,2,4-triazin-5(4H)-one ${ }^{32}$, Keeping this in mind we have subsequently carried out the synthesis of s-triazine based 4-(benzo[ $d]$ thiazol-2yl)aniline and 6-(4-methoxyphenyl)-4-phenyl-2H-1,3oxazin-2-amine derivatives to explore the synthesis of more potential bioactive molecules in one framework.

\section{Methods and Materials}

All reactions except those in aqueous media were carried out by standard techniques for the exclusion of moisture. Melting points were determined on an electro thermal melting point apparatus and are reported uncorrected. TLC on silica gel plates were used for purity checking and reaction monitoring. Elemental analysis $(\% \mathrm{C}, \mathrm{H}, \mathrm{N})$ was carried out by a 
Perkin-Elmer $2400 \mathrm{CHN}$ analyzer. IR spectra of all compounds were recorded on a Perkin-Elmer FT-IR spectrophotometer in $\mathrm{KBr}$. ${ }^{1} \mathrm{HNMR}$ spectra were recorded on Bruker Avance II-400 $\mathrm{MHz}$ and ${ }^{13}$ CNMR spectra on Bruker Avance II-400, $100 \mathrm{MHz}$ in DMSO- $d 6$ as a solvent and tetramethylsilane (TMS) as an internal standard. Mass spectra were recorded on triple quadrapole LCMS-6410 from Agilent Technology.

Preparation of 1-(4, 6-dichloro-1,3,5-triazin-2-yl)-3phenylthiourea: (T1 to T15)

To the stirred solution of cyanuric chloride $(0.01$ mol) in acetone $(25 \mathrm{~mL})$ at $0-5{ }^{\circ} \mathrm{C}$, the solution of substituted phenyl thiourea $(0.01 \mathrm{~mol})$ in acetone $(15$ $\mathrm{mL}$ ) was added and $\mathrm{pH}$ was maintained neutral by the addition of $10 \%$ sodium bi-carbonate solution from time to time as per requirement of reaction condition. The stirring was continued at $0-5^{\circ} \mathrm{C}$ for 2 hours. After the completion of reaction the stirring was stopped and the solution was treated with crushed ice. The solid product obtained was filtered and dried. The crude product was purified by crystallization from absolute alcohol to get title compound.

Preparation of 1-(4-chloro-6-(2-(4-nitrobenzoyl) hydrazinyl)-1, 3, 5-triazin-2-yl)-3-phenyl thiourea:(T1 to $15 \mathrm{G}$ )

\section{Reaction Scheme \\ Step 1:}

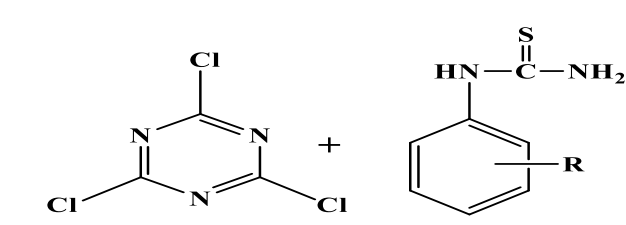

2,4,6-Trichloro-1,3,5-triazine 1-Phenylthiourea

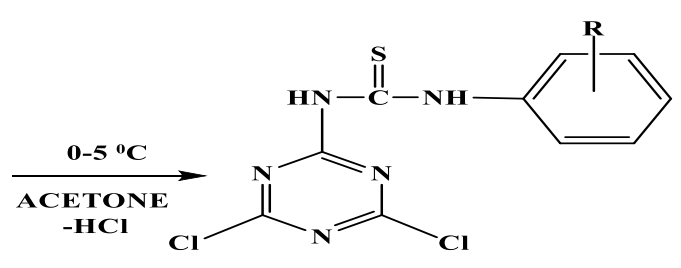

1-(4,6-Dichloro-1,3,5-triazin-2-yl)-3-phenylthiourea
To a stirred solution of (T1 to 15$)(0.01 \mathrm{~mol})$ in DMF $(25 \mathrm{~mL})$, the solution of 4-nitrobenzohydrazide (0.01 mol) in DMF $(15 \mathrm{~mL})$ was added drop wise maintaining the temperature at $40{ }^{\circ} \mathrm{C}$, the $\mathrm{pH}$ was maintained neutral by the addition of $10 \%$ sodium bicarbonate solution from time to time as per requirement of reaction condition. The temperature was gradually raised to $45^{\circ} \mathrm{C}$ during three hours. After the completion of reaction, the resultant content was poured into icecold water. The solid product obtained was filtered and dried. The crude product was purified by crystallization from absolute alcohol to get the title compound.

Preparation of 1-(4-((6-(4-methoxyphenyl)-4-phenyl6H-1, 3-thiazin-2-yl) amino)-6-(2-(4-nitrobenzoyl) hydrazinyl)-1, 3, 5-triazin-2-yl)-3-phenylthiourea: (T1 to $15 \mathrm{GE}$ )

A mixture of (T1G to $15 \mathrm{G})(0.01 \mathrm{~mol})$ and $6-(4-$ methoxyphenyl)-4-phenyl-6H-1, 3-thiazin-2-amine $(0.01 \mathrm{~mol})$ in DMF $(15 \mathrm{~mL})$ was refluxed in oil bath. The temperature was gradually raised to $80-100{ }^{\circ} \mathrm{C}$ during four hours, the $\mathrm{pH}$ being maintained neutral by the addition of $10 \%$ sodium bi-carbonate solution from time to time as per requirement of reaction condition. After the completion of reaction charcoal was added in R.B.F. and heated then mixture was filtered into cold water. The solid product obtained was filtered and dried. The crude product was purified by recrystallization from absolute alcohol.

\section{Step 2: (T1 to 15)}

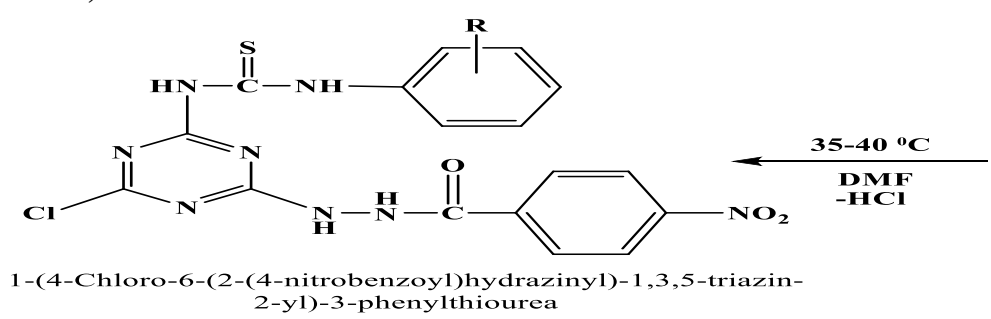

(T1 to $15 \mathrm{G}$ ) 
Step 3:

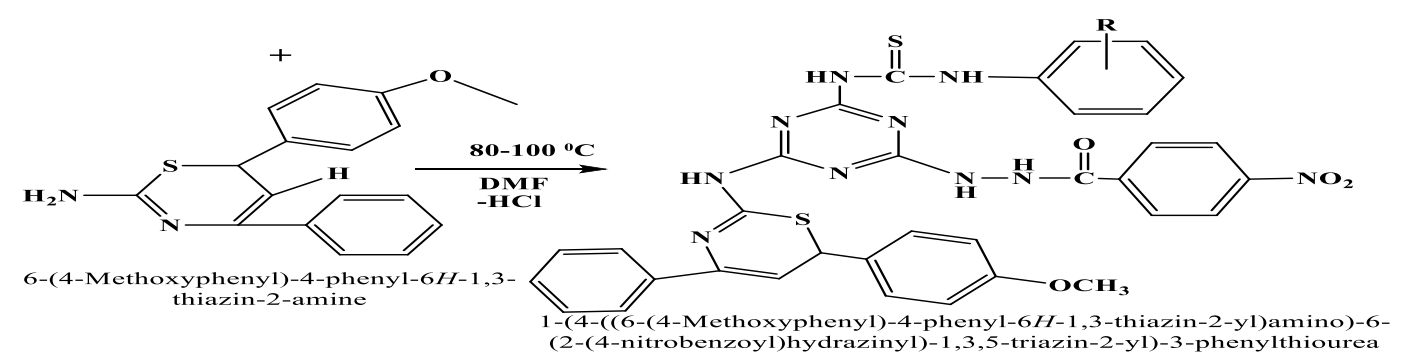

(E)

(T1 to 15GE)

Compound TlGE: $\mathrm{IR}\left(\mathrm{KBr}, \mathrm{cm}^{-1}\right)$ : $-\mathrm{C}=\mathrm{N}$ str. in s-triazine (783.3), $\mathrm{C}-\mathrm{S}-\mathrm{C}$ str. in thiazole (830.5), $-\mathrm{C}=\mathrm{S}$ str. in thiourea (1148.0), - $\mathrm{N}=\mathrm{O}-$ str. as $-\mathrm{NO}_{2}(1540.0)$, $-\mathrm{C}=\mathrm{O}$ str. in amide (1591.0), $-\mathrm{N}-\mathrm{H}$ deformation in $-2^{0} \mathrm{NH}(1700.0)$, $-\mathrm{C}-\mathrm{H}$ str. in $-\mathrm{OCH}_{3}(2831.4)$, $-\mathrm{C}-\mathrm{H}$ str. in aromatic $(3173.2),-\mathrm{N}-\mathrm{H}$ str. in $-2^{0} \mathrm{NH}(3384.1) .{ }^{1} \mathrm{H}$ NMR $\left(400.0 \mathrm{MHz}, \mathrm{DMSO}-\mathrm{d}_{6}, \delta_{\mathrm{H}}\right.$ ppm): 10.61 (s, 2H, -CS-NH), 9.11 (s, 1H, -CO-NH), 8.24 (s, 1H, -NH-NH-C), 6.90-8.32 (m, 18H, Ar), 6.88 (s, 1H, $\mathrm{Ar}=\mathrm{C}-\mathrm{H}), 5.11(\mathrm{~s}, 1 \mathrm{H}, \mathrm{Ar}-\mathrm{S}-\mathrm{CH}), 3.91(\mathrm{~s}, 1 \mathrm{H},-\mathrm{NH}), 3.70-3.80\left(\mathrm{~s}, 3 \mathrm{H},-\mathrm{OCH}_{3}\right)$.

Compound T2GE: -C=N str. in s-triazine (785.1),-C-S-C- str. in thiazine (861.1), -C=S str. in thiourea (1121), -C$\mathrm{CH}_{3}$ str. in aromatic ring(1330.1), $-\mathrm{N}=\mathrm{O}$ str. in aromatic ring (1537), $-\mathrm{C}=\mathrm{O}-$ str. in amide(1606.7), $-\mathrm{N}-\mathrm{H}$ deformation in $-2^{0} \mathrm{NH}(1718.5)$, $-\mathrm{C}-\mathrm{H}$ str. in $-\mathrm{OCH}_{3}(2815.1)$, $-\mathrm{C}-\mathrm{H}$ str. in $-\mathrm{CH}_{3}$ (2924.1), $-\mathrm{C}-\mathrm{H}$ str. in aromatic (3136.5), -N-H str. in $-2^{0} \mathrm{NH}(3400.1) .{ }^{1} \mathrm{H}$ NMR $\left(400.0 \mathrm{MHz}, \mathrm{DMSO}_{6} \mathrm{~d}_{6}, \delta_{\mathrm{H}} \mathrm{ppm}\right): 10.68$ (s, 2H, -CS-NH), 9.01 (s, $\left.1 \mathrm{H},-\mathrm{CO}-\mathrm{NH}\right), 8.19$ (s, 1H, -NH-NH-C), 6.92-8.10 (m, 17H, Ar), 6.90(s, 1H, Ar=C-H), $5.07(\mathrm{~s}, 1 \mathrm{H}, \mathrm{Ar}-\mathrm{S}-\mathrm{CH}), 3.93(\mathrm{~s}, 1 \mathrm{H},-\mathrm{NH}), 3.80-$

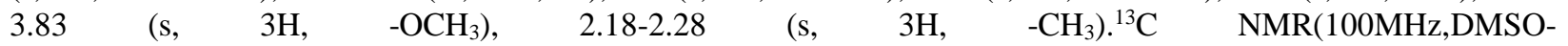
$\left.\mathrm{d}_{6}, \delta_{\mathrm{C}} \mathrm{ppm}\right): 18.23,35.14,55.23,113.84,114.26(\mathrm{db}), 125.77,127.2(\mathrm{db}), 127.72,128.21(\mathrm{db}), 128.26,128.52,128.63$ (db),130.01(db),130.57(db),132.64(db),133.40,134.24,136.08,137.84,143.94,151.35,159.3(db),165.25(db), 172.52,179.21(db).MS (EI): m/z: $718.5(\mathrm{M}+)$.

Table 1: Physicochemical data of the synthesized compounds T1GE to T15GE

\begin{tabular}{|c|c|c|c|c|c|c|c|}
\hline \multirow[t]{2}{*}{ S. No. } & \multirow[t]{2}{*}{$\mathbf{R}$} & \multirow[t]{2}{*}{ M.P. ${ }^{\circ} \mathrm{C}$} & \multirow{2}{*}{$\begin{array}{c}\text { Yield } \\
\%\end{array}$} & \multirow[t]{2}{*}{ Mol. Formula } & \multicolumn{3}{|c|}{$\begin{array}{l}\text { Calculated } \\
\text { (Found) \% }\end{array}$} \\
\hline & & & & & $\mathrm{C}$ & $\mathbf{H}$ & $\mathbf{N}$ \\
\hline T1GE & $\mathrm{H}$ & 190 & 70.25 & $\mathrm{C}_{34} \mathrm{H}_{28} \mathrm{~N}_{10} \mathrm{O}_{4} \mathrm{~S}_{2}$ & 57.94(57.90) & $4.00(3.98)$ & $19.87(19.81)$ \\
\hline T2GE & $2-\mathrm{CH}_{3}$ & 205 & 55.45 & $\mathrm{C}_{35} \mathrm{H}_{30} \mathrm{~N}_{10} \mathrm{O}_{4} \mathrm{~S}_{2}$ & $58.48(58.42)$ & $4.21(4.16)$ & $19.49(19.45)$ \\
\hline T3GE & $4-\mathrm{CH}_{3}$ & 213 & 60.25 & $\mathrm{C}_{35} \mathrm{H}_{30} \mathrm{~N}_{10} \mathrm{O}_{4} \mathrm{~S}_{2}$ & $58.48(58.46)$ & $4.21(4.13)$ & $19.49(19.44)$ \\
\hline T4GE & $2-\mathrm{OCH}_{3}$ & 210 & 65.70 & $\mathrm{C}_{35} \mathrm{H}_{30} \mathrm{~N}_{10} \mathrm{O}_{5} \mathrm{~S}_{2}$ & $57.21(57.16)$ & $4.12(4.08)$ & $19.06(19.01)$ \\
\hline T5GE & $4-\mathrm{OCH}_{3}$ & 185 & 69.55 & $\mathrm{C}_{35} \mathrm{H}_{30} \mathrm{~N}_{10} \mathrm{O}_{5} \mathrm{~S}_{2}$ & $57.21(57.18)$ & $4.12(4.10)$ & $19.06(19.03)$ \\
\hline T6GE & $3-\mathrm{NO}_{2}$ & 190 & 55.55 & $\mathrm{C}_{34} \mathrm{H}_{27} \mathrm{~N}_{11} \mathrm{O}_{6} \mathrm{~S}_{2}$ & $54.47(54.43)$ & $3.63(3.60)$ & $20.55(20.50)$ \\
\hline T7GE & $3-\mathrm{Cl}$ & 200 & 58.60 & $\mathrm{C}_{34} \mathrm{H}_{27} \mathrm{ClN}_{10} \mathrm{O}_{4} \mathrm{~S}_{2}$ & $55.24(55.20)$ & $3.68(3.62)$ & $18.95(18.90)$ \\
\hline T8GE & 4-Cl & 165 & 71.15 & $\mathrm{C}_{34} \mathrm{H}_{27} \mathrm{ClN}_{10} \mathrm{O}_{4} \mathrm{~S}_{2}$ & $55.24(55.21)$ & $3.68(3.66)$ & $18.95(18.88)$ \\
\hline T9GE & $4-\mathrm{F}$ & 188 & 60.50 & $\mathrm{C}_{34} \mathrm{H}_{27} \mathrm{FN}_{10} \mathrm{O}_{4} \mathrm{~S}_{2}$ & $56.50(56.48)$ & $3.77(3.72)$ & $19.38(19.36)$ \\
\hline T10GE & $4-\mathrm{Br}$ & 170 & 68.60 & $\mathrm{C}_{34} \mathrm{H}_{27} \mathrm{BrN}_{10} \mathrm{O}_{4} \mathrm{~S}_{2}$ & $52.11(52.09)$ & $3.47(3.44)$ & $17.87(17.81)$ \\
\hline T11GE & $2-\mathrm{NO}_{2}$ & 155 & 70.25 & $\mathrm{C}_{34} \mathrm{H}_{27} \mathrm{~N}_{11} \mathrm{O}_{6} \mathrm{~S}_{2}$ & $54.47(54.44)$ & $3.63(3.61)$ & $20.55(20.52)$ \\
\hline T12GE & $3-\mathrm{OCH}_{3}$ & 220 & 67.80 & $\mathrm{C}_{35} \mathrm{H}_{30} \mathrm{~N}_{10} \mathrm{O}_{5} \mathrm{~S}_{2}$ & $57.21(57.17)$ & $4.12(4.10)$ & $19.06(19.04)$ \\
\hline T13GE & $3-\mathrm{CH}_{3}$ & 185 & 62.65 & $\mathrm{C}_{35} \mathrm{H}_{30} \mathrm{~N}_{10} \mathrm{O}_{4} \mathrm{~S}_{2}$ & $58.48(58.43)$ & $4.21(4.17)$ & $19.49(19.44)$ \\
\hline T14GE & $4-\mathrm{NO}_{2}$ & 215 & 66.75 & $\mathrm{C}_{34} \mathrm{H}_{27} \mathrm{~N}_{11} \mathrm{O}_{6} \mathrm{~S}_{2}$ & $54.47(54.42)$ & $3.63(3.58)$ & $20.55(20.52)$ \\
\hline T15GE & -Naphthyl & 225 & 69.85 & $\mathrm{C}_{38} \mathrm{H}_{30} \mathrm{~N}_{10} \mathrm{O}_{4} \mathrm{~S}_{2}$ & $60.47(60.42)$ & $4.01(3.99)$ & $18.56(18.53)$ \\
\hline
\end{tabular}

Compound T3GE: $\mathrm{IR}\left(\mathrm{KBr}, \mathrm{cm}^{-1}\right)$ : $-\mathrm{C}=\mathrm{N}$ str. in striazine (786.3), $-\mathrm{C}-\mathrm{S}-\mathrm{C}$ str. in thiazole $(830.5),-\mathrm{C}=\mathrm{S}$ str. in thiourea (1143.0), $-\mathrm{C}_{-} \mathrm{CH}_{3}$ str. in aromatic ring(1348.0), $-\mathrm{N}=\mathrm{O}-$ str. as $-\mathrm{NO}_{2}(1532.0),-\mathrm{C}=\mathrm{O}$ str. in amide(1596.0), - $\mathrm{N}-\mathrm{H}$ deformation in $-2^{0} \mathrm{NH}(1712.0)$, $\mathrm{C}-\mathrm{H}$ str. in $-\mathrm{OCH}_{3}(2843.4)$, $-\mathrm{C}-\mathrm{H}$ str. in aromatic (3180.2), $-\mathrm{N}-\mathrm{H}$ str. in $-2^{0} \mathrm{NH}(3388.1) .{ }^{1} \mathrm{H}$ NMR $(400.0$ MHz, DMSO-d 6 , $\left.\delta_{\mathrm{H}} \mathrm{ppm}\right): 10.61$ (s, $\left.2 \mathrm{H},-\mathrm{CS}-\mathrm{NH}\right), 9.12$ (s, 1H, -CO-NH), 8.31 (s, 1H, -NH-NH-C), 6.98-8.23 (m, 17H, Ar), 6.90 (s, 1H, Ar=C-H), 5.13 (s, 1H, Ar-S-
$\mathrm{CH}), 3.99$ (s, 1H, -NH), 3.65-3.88 (s, 3H, $\left.-\mathrm{OCH}_{3}\right), 2.14-$ $2.20\left(\mathrm{~s}, 3 \mathrm{H},-\mathrm{CH}_{3}\right)$.

Compound T4GE: $\mathrm{IR}\left(\mathrm{KBr}, \mathrm{cm}^{-1}\right)$ : $-\mathrm{C}=\mathrm{N}$ str. in striazine (789.3),-C-S-C str. in thiazole (839.5), $-\mathrm{C}=\mathrm{S}$ str. in thiourea (1149.0), $-\mathrm{N}=\mathrm{O}-$ str. as $-\mathrm{NO}_{2}(1536.0)$, $\mathrm{C}=\mathrm{O}$ str. in amide (1594.0), $-\mathrm{N}-\mathrm{H}$ deformation in $-2^{0}$ $\mathrm{NH}(1701.0)$, - $\mathrm{C}-\mathrm{H}$ str. in $-\mathrm{OCH}_{3}(2838.4)$, $-\mathrm{C}-\mathrm{H}$ str. in aromatic (3177.2), $-\mathrm{N}-\mathrm{H}$ str. in $-2^{0} \mathrm{NH}(3382.1) .{ }^{1} \mathrm{H}$ NMR (400.0 MHz, DMSO-d 6 , $\left.\delta_{\mathrm{H}} \mathrm{ppm}\right): 10.76$ (s, 2H, CS-NH), 9.16 (s, 1H, -CO-NH), 8.25 (s, 1H, -NH-NH- 
C), 6.84-8.11 (m, 17H, Ar), 6.78(s, 1H, Ar=C-H), 5.22 (s, 1H, Ar-S-CH), 3.89 (s, 1H, -NH), 3.70-3.81 (s, 6H, $\left.\mathrm{OCH}_{3}\right)$.

Compound T5GE: IR $\left(\mathrm{KBr}, \mathrm{cm}^{-1}\right):-\mathrm{C}=\mathrm{N}$ str. in striazine (780.3), $-\mathrm{C}-\mathrm{S}-\mathrm{C}$ str. in thiazole $(832.1),-\mathrm{C}=\mathrm{S}$ str. in thiourea (1111.0), - $\mathrm{N}=\mathrm{O}-$ str. as $-\mathrm{NO}_{2}(1545.0)$, $\mathrm{C}=\mathrm{O}$ str. in amide (1595.0), $-\mathrm{N}-\mathrm{H}$ deformation in $-2^{0}$ $\mathrm{NH}(1709.0)$, $-\mathrm{C}-\mathrm{H}$ str. in $-\mathrm{OCH}_{3}(2842.4)$, $-\mathrm{C}-\mathrm{H}$ str. in aromatic (3188.2), - $\mathrm{N}-\mathrm{H}$ str. in $-2^{0} \mathrm{NH}(3380.1) .{ }^{1} \mathrm{H}$ NMR $\left(400.0 \mathrm{MHz}\right.$, DMSO-d 6 , $\left.\delta_{\mathrm{H}} \mathrm{ppm}\right): 10.60(\mathrm{~s}, 2 \mathrm{H},-$ CS-NH), $9.12(\mathrm{~s}, 1 \mathrm{H},-\mathrm{CO}-\mathrm{NH}), 8.18(\mathrm{~s}, 1 \mathrm{H},-\mathrm{NH}-\mathrm{NH}-$ C), 6.71-8.08 (m, 17H, Ar), 6.89(s, 1H, Ar=C-H), 5.11 (s, 1H, Ar-S-CH), 3.91 (s, 1H, -NH), 3.65-3.88 (s, 6H, $\left.\mathrm{OCH}_{3}\right)$.

Compound T6GE: $\mathrm{IR}\left(\mathrm{KBr}, \mathrm{cm}^{-1}\right)$ : $-\mathrm{C}=\mathrm{N}$ str. in striazine (780.3), $-\mathrm{C}-\mathrm{S}-\mathrm{C}$ str. in thiazole (824.5), $-\mathrm{C}=\mathrm{S}$ str. in thiourea (1135.0), $-\mathrm{N}=\mathrm{O}$ - str. as $-\mathrm{NO}_{2}(1545.0)$, $\mathrm{C}=\mathrm{O}$ str. in amide $(1600.0),-\mathrm{N}-\mathrm{H}$ deformation in $-2^{0}$ $\mathrm{NH}(1711.0)$, $-\mathrm{C}-\mathrm{H}$ str. in $-\mathrm{OCH}_{3}(2828.4)$, $-\mathrm{C}-\mathrm{H}$ str. in aromatic (3178.2), - $\mathrm{N}-\mathrm{H}$ str. in $-2^{0} \mathrm{NH}(3380.1) .{ }^{1} \mathrm{H}$ NMR (400.0 MHz, DMSO-d $\left.\mathrm{d}_{6}, \delta_{\mathrm{H}} \mathrm{ppm}\right): 10.55(\mathrm{~s}, 2 \mathrm{H},-$ CS-NH), 9.14 (s, 1H, -CO-NH), 8.20 (s, 1H, -NH-NHC), 6.78-8.24 (m, 17H, Ar), 6.90(s, $1 \mathrm{H}, \mathrm{Ar}=\mathrm{C}-\mathrm{H}), 5.23$ (s, 1H, Ar-S-CH), 3.98 (s, 1H, -NH), 3.61-3.91 (s, 3H, $\left.\mathrm{OCH}_{3}\right)$.

Compound T7GE: IR $\left(\mathrm{KBr}, \mathrm{cm}^{-1}\right)$ : $-\mathrm{C}-\mathrm{Cl}$ str. In aromatic ring (753.0), $-\mathrm{C}=\mathrm{N}$ str. in s-triazine (778.3), $-\mathrm{C}$ $\mathrm{S}-\mathrm{C}$ str. in thiazole $(826.5),-\mathrm{C}=\mathrm{S}$ str. in thiourea (1145.0), $-\mathrm{N}=\mathrm{O}-$ str. as $-\mathrm{NO}_{2}(1531.0),-\mathrm{C}=\mathrm{O}$ str. in amide(1580.0), - $\mathrm{N}-\mathrm{H}$ deformation in $-2^{0} \mathrm{NH}(1716.0)$, $\mathrm{C}-\mathrm{H}$ str. in $-\mathrm{OCH}_{3}(2839.4)$, $-\mathrm{C}-\mathrm{H}$ str. in aromatic (3175.2), $-\mathrm{N}-\mathrm{H}$ str. in $-2^{0} \mathrm{NH}(3388.1) .{ }^{1} \mathrm{H}$ NMR $(400.0$ MHz, DMSO-d $\left.{ }_{6}, \delta_{\mathrm{H}} \mathrm{ppm}\right): 10.61$ (s, 2H, -CS-NH), 9.11 (s, 1H, -CO-NH), 8.27 (s, 1H, -NH-NH-C), 6.90-8.11 $(\mathrm{m}, 17 \mathrm{H}, \mathrm{Ar}), 6.95(\mathrm{~s}, 1 \mathrm{H}, \mathrm{Ar}=\mathrm{C}-\mathrm{H}), 5.12(\mathrm{~s}, 1 \mathrm{H}, \mathrm{Ar}-\mathrm{S}-$ $\mathrm{CH}), 3.90(\mathrm{~s}, 1 \mathrm{H},-\mathrm{NH}), 3.70-3.86\left(\mathrm{~s}, 3 \mathrm{H},-\mathrm{OCH}_{3}\right)$.

Compound T8GE: IR $\left(\mathrm{KBr}, \mathrm{cm}^{-1}\right)$ : $-\mathrm{C}-\mathrm{Cl}$ str. In aromatic ring (751.0), $-\mathrm{C}=\mathrm{N}$ str. in s-triazine (781.3),$\mathrm{C}-\mathrm{S}-\mathrm{C}$ str. in thiazole (835), $-\mathrm{C}=\mathrm{S}$ str. in thiourea (1128.0), - $\mathrm{N}=\mathrm{O}-$ str. as $-\mathrm{NO}_{2}(1531.0),-\mathrm{C}=\mathrm{O}$ str. in amide(1599.0), - $\mathrm{N}-\mathrm{H}$ deformation in $-2^{0} \mathrm{NH}(1725.0)$, C-H str. in $-\mathrm{OCH}_{3}(2846.4),-\mathrm{C}-\mathrm{H}$ str. in aromatic (3171.2), $-\mathrm{N}-\mathrm{H}$ str. in $-2^{0} \mathrm{NH}(3389.1) .{ }^{1} \mathrm{H}$ NMR (400.0 $\left.\mathrm{MHz}, \mathrm{DMSO}_{\mathrm{d}}, \delta_{\mathrm{H}} \mathrm{ppm}\right): 10.61$ (s, $\left.2 \mathrm{H},-\mathrm{CS}-\mathrm{NH}\right), 9.43$ (s, 1H, -CO-NH), 8.11 (s, 1H, -NH-NH-C), 6.71-8.45 $(\mathrm{m}, 17 \mathrm{H}, \mathrm{Ar}), 6.90(\mathrm{~s}, 1 \mathrm{H}, \mathrm{Ar}=\mathrm{C}-\mathrm{H}), 5.15(\mathrm{~s}, 1 \mathrm{H}, \mathrm{Ar}-\mathrm{S}-$ $\mathrm{CH}), 3.99(\mathrm{~s}, 1 \mathrm{H},-\mathrm{NH}), 3.71-3.81\left(\mathrm{~s}, 3 \mathrm{H},-\mathrm{OCH}_{3}\right)$.

Compound T9GE: IR $\left(\mathrm{KBr}, \mathrm{cm}^{-1}\right)$ : $-\mathrm{C}=\mathrm{N}$ str. in striazine (787.3),-C-S-C str. in thiazole (831.5), -C-F str. In aromatic ring(1097.0), $-\mathrm{C}=\mathrm{S}$ str. in thiourea (1140.0), $-\mathrm{N}=\mathrm{O}-\quad$ str. as $-\mathrm{NO}_{2}(1536.0), \quad-\mathrm{C}=\mathrm{O} \quad$ str. in amide(1594.0), - $\mathrm{N}-\mathrm{H}$ deformation in $-2^{0} \mathrm{NH}(1701.0)$, $\mathrm{C}-\mathrm{H}$ str. in $-\mathrm{OCH}_{3}(2838.4),-\mathrm{C}-\mathrm{H}$ str. in aromatic
(3177.2), - $\mathrm{N}-\mathrm{H}$ str. in $-2^{0} \mathrm{NH}(3382.1) .{ }^{1} \mathrm{H}$ NMR $(400.0$ MHz, DMSO-d 6 , $\delta_{\mathrm{H}} \mathrm{ppm}$ ): 10.67 (s, 2H, -CS-NH), 9.02 (s, 1H, -CO-NH), 8.21 (s, 1H, -NH-NH-C), 6.98-8.38 $(\mathrm{m}, 17 \mathrm{H}, \mathrm{Ar}), 6.91(\mathrm{~s}, 1 \mathrm{H}, \mathrm{Ar}=\mathrm{C}-\mathrm{H}), 5.04(\mathrm{~s}, 1 \mathrm{H}, \mathrm{Ar}-\mathrm{S}-$ $\mathrm{CH}), 3.93(\mathrm{~s}, 1 \mathrm{H},-\mathrm{NH}), 3.75-3.82\left(\mathrm{~s}, 3 \mathrm{H},-\mathrm{OCH}_{3}\right)$.

Compound TI0GE: IR $\left(\mathrm{KBr}, \mathrm{cm}^{-1}\right)$ : $-\mathrm{C}=\mathrm{N}$ str. in striazine (780.3),-C-S-C str. in thiazole (826.5), $-\mathrm{C}-\mathrm{Br}$ str. In aromatic $\operatorname{ring}(1097.0),-C=S$ str. in thiourea (1149.0), $-\mathrm{N}=\mathrm{O}$ - str. as $-\mathrm{NO}_{2}(1543.0),-\mathrm{C}=\mathrm{O}$ str. in amide(1595.0), - $\mathrm{N}-\mathrm{H}$ deformation in $-2^{0} \mathrm{NH}(1712.0)$, $\mathrm{C}-\mathrm{H}$ str. in $-\mathrm{OCH}_{3}(2839.4),-\mathrm{C}-\mathrm{H}$ str. in aromatic (3179.2), - $\mathrm{N}-\mathrm{H}$ str. in $-2^{0} \mathrm{NH}(3380.1) .{ }^{1} \mathrm{H}$ NMR $(400.0$ MHz, DMSO-d $\mathrm{d}_{6}, \delta_{\mathrm{H}} \mathrm{ppm}$ ): 10.66 (s, 2H, -CS-NH), 9.11 (s, 1H, -CO-NH), 8.27 (s, 1H, -NH-NH-C), 6.74-8.22 $(\mathrm{m}, 17 \mathrm{H}, \mathrm{Ar}), 6.76(\mathrm{~s}, 1 \mathrm{H}, \mathrm{Ar}=\mathrm{C}-\mathrm{H}), 5.13(\mathrm{~s}, 1 \mathrm{H}, \mathrm{Ar}-\mathrm{S}-$ $\mathrm{CH}), 3.98(\mathrm{~s}, 1 \mathrm{H},-\mathrm{NH}), 3.70-3.84\left(\mathrm{~s}, 3 \mathrm{H},-\mathrm{OCH}_{3}\right)$.

Compound TllGE: $\operatorname{IR}\left(\mathrm{KBr}, \mathrm{cm}^{-1}\right)$ : $-\mathrm{C}=\mathrm{N}$ str. in striazine (781.3), $-\mathrm{C}-\mathrm{S}-\mathrm{C}$ str. in thiazole $(826.5),-\mathrm{C}=\mathrm{S}$ str. in thiourea (1132.0), $-\mathrm{N}=\mathrm{O}$ - str. as $-\mathrm{NO}_{2}(1523.0)$, $\mathrm{C}=\mathrm{O}$ str. in amide(1599.0), $-\mathrm{N}-\mathrm{H}$ deformation in $-2^{0}$ $\mathrm{NH}(1723.0)$, $-\mathrm{C}-\mathrm{H}$ str. in $-\mathrm{OCH}_{3}(2830.4)$, $-\mathrm{C}-\mathrm{H}$ str. in aromatic $(3170.2),-\mathrm{N}-\mathrm{H}$ str. in $-2^{0} \mathrm{NH}(3391.1) .{ }^{1} \mathrm{H}$ NMR (400.0 MHz, DMSO-d $\left.{ }_{6}, \delta_{\mathrm{H}} \mathrm{ppm}\right): 10.54$ (s, 2H, CS-NH), 9.16 (s, 1H, -CO-NH), 8.43 (s, 1H, -NH-NHC), 6.50-8.18 (m, 17H, Ar), $6.76(\mathrm{~s}, 1 \mathrm{H}, \mathrm{Ar}=\mathrm{C}-\mathrm{H}), 5.13$ (s, 1H, Ar-S-CH), 3.87 (s, 1H, -NH), 3.81-3.89 (s, 3H, $\left.\mathrm{OCH}_{3}\right)$.

Compound T12GE: $\operatorname{IR}\left(\mathrm{KBr}, \mathrm{cm}^{-1}\right):-\mathrm{C}=\mathrm{N}$ str. in striazine (775.3), $-\mathrm{C}-\mathrm{S}-\mathrm{C}$ str. in thiazole (823.5), $-\mathrm{C}=\mathrm{S}$ str. in thiourea $(1154.0),-\mathrm{N}=\mathrm{O}$ - str. as $-\mathrm{NO}_{2}(1541.0)$, $\mathrm{C}=\mathrm{O}$ str. in amide(1576.0), $-\mathrm{N}-\mathrm{H}$ deformation in $-2^{0}$ $\mathrm{NH}(1719.0)$, -C-H str. in $-\mathrm{OCH}_{3}(2813.4)$, $-\mathrm{C}-\mathrm{H}$ str. in aromatic (3165.2), $-\mathrm{N}-\mathrm{H}$ str. in $-2^{0} \mathrm{NH}(3311.1) .{ }^{1} \mathrm{H}$ NMR (400.0 MHz, DMSO-d $\left.{ }_{6}, \delta_{\mathrm{H}} \mathrm{ppm}\right): 10.55$ (s, 2H, CS-NH), 9.17 (s, 1H, -CO-NH), 8.11 (s, 1H, -NH-NHC), 6.78-8.45 (m, 17H, Ar), $6.76(\mathrm{~s}, 1 \mathrm{H}, \mathrm{Ar}=\mathrm{C}-\mathrm{H}), 5.07$ (s, 1H, Ar-S-CH), 3.91 (s, 1H, -NH), 3.77-3.87 (s, 6H, $\left.\mathrm{OCH}_{3}\right)$.

Compound T13GE: $\mathrm{IR}\left(\mathrm{KBr}, \mathrm{cm}^{-1}\right)$ : $-\mathrm{C}=\mathrm{N}$ str. in striazine (792.3), $-\mathrm{C}-\mathrm{S}-\mathrm{C}$ str. in thiazole (826.5), $-\mathrm{C}=\mathrm{S}$ str. in thiourea (1146.0), $-\mathrm{N}=\mathrm{O}-$ str. as $-\mathrm{NO}_{2}(1541.0)$, $\mathrm{C}=\mathrm{O}$ str. in amide(1595.0), $-\mathrm{N}-\mathrm{H}$ deformation in $-2^{0}$ $\mathrm{NH}(1712.0)$, -C-H str. in $-\mathrm{OCH}_{3}(2845.4)$, $-\mathrm{C}-\mathrm{H}$ str. in aromatic (3188.2), - $\mathrm{N}-\mathrm{H}$ str. in $-2^{0} \mathrm{NH}(3391.1) .{ }^{1} \mathrm{H}$ NMR (400.0 MHz, DMSO-d 6 , $\left.\delta_{\mathrm{H}} \mathrm{ppm}\right): 10.71$ (s, 2H, CS-NH), 9.15 (s, 1H, -CO-NH), 8.11 (s, 1H, -NH-NHC), 6.71-8.16 (m, 17H, Ar), 6.67(s, 1H, Ar=C-H), 4.96 (s, 1H, Ar-S-CH), 3.99 (s, 1H, -NH), 3.79-3.91 (s, 3H, $\left.\mathrm{OCH}_{3}\right), 2.17-2.31\left(\mathrm{~s}, 3 \mathrm{H},-\mathrm{CH}_{3}\right)$.

Compound T14GE: $\operatorname{IR}\left(\mathrm{KBr}, \mathrm{cm}^{-1}\right)$ : $-\mathrm{C}=\mathrm{N}$ str. in striazine (795.3), $-\mathrm{C}-\mathrm{S}-\mathrm{C}$ str. in thiazole (822.5), $-\mathrm{C}=\mathrm{S}$ str. in thiourea (1144.0), $-\mathrm{N}=\mathrm{O}-$ str. as $-\mathrm{NO}_{2}(1541.0)$, $\mathrm{C}=\mathrm{O}$ str. in amide(1586.0), $-\mathrm{N}-\mathrm{H}$ deformation in $-2^{0}$ 
$\mathrm{NH}(1696.0)$, $-\mathrm{C}-\mathrm{H}$ str. in $-\mathrm{OCH}_{3}(2827.4)$, $-\mathrm{C}-\mathrm{H}$ str. in aromatic (3165.2), $-\mathrm{N}-\mathrm{H}$ str. in $-2^{0} \mathrm{NH}(3380.1) .{ }^{1} \mathrm{H}$ NMR (400.0 MHz, DMSO-d $\left.\mathrm{d}_{6}, \delta_{\mathrm{H}} \mathrm{ppm}\right): 10.76$ (s, 2H, CS-NH), 9.17 (s, 1H, -CO-NH), 8.29 (s, 1H, -NH-NHC), 6.90-8.45 (m, 17H, Ar), $6.84(\mathrm{~s}, 1 \mathrm{H}, \mathrm{Ar}=\mathrm{C}-\mathrm{H}), 5.18$ (s, 1H, Ar-S-CH), 3.84 (s, 1H, -NH), 3.67-3.97 (s, 3H, $\left.\mathrm{OCH}_{3}\right)$.

Compound T15GE: $\mathrm{IR}\left(\mathrm{KBr}, \mathrm{cm}^{-1}\right)$ : $-\mathrm{C}=\mathrm{N}$ str. in striazine (796.3),-C-S-C str. in thiazole (842.5), $-\mathrm{C}=\mathrm{S}$ str. in thiourea (1156.0), $-\mathrm{N}=\mathrm{O}-$ str. as $-\mathrm{NO}_{2}(1543.0)$, $\mathrm{C}=\mathrm{O}$ str. in amide(1578.0), $-\mathrm{N}-\mathrm{H}$ deformation in $-2^{0}$ $\mathrm{NH}(1706.0)$, -C-H str. in $-\mathrm{OCH}_{3}(2825.4)$, $-\mathrm{C}-\mathrm{H}$ str. in aromatic (3167.2), $-\mathrm{N}-\mathrm{H}$ str. in $-2^{0} \mathrm{NH}(3374.1) .{ }^{1} \mathrm{H}$ NMR (400.0 MHz, DMSO-d 6 , $\left.\delta_{\mathrm{H}} \mathrm{ppm}\right): 10.65$ (s, 2H, CS-NH), 8.94 (s, 1H, -CO-NH), 8.11 (s, 1H, -NH-NHC), 6.76-8.30 (m, 20H, Ar), $6.88(\mathrm{~s}, 1 \mathrm{H}, \mathrm{Ar}=\mathrm{C}-\mathrm{H}), 5.14$ (s, 1H, Ar-S-CH), 3.87 (s, 1H, -NH), 3.70-3.80 (s, 3H, $\left.\mathrm{OCH}_{3}\right)$.

Table 2

\begin{tabular}{|c|c|c|c|c|c|c|c|c|c|}
\hline \multirow{3}{*}{ S.N. } & \multirow{3}{*}{ Comp. } & \multirow{3}{*}{$\mathbf{R}=$} & \multicolumn{7}{|c|}{ Minimum Inhibitory Concentration $(\mu \mathrm{g} / \mathrm{mL})$} \\
\hline & & & \multicolumn{2}{|c|}{ Gram Negative Bacteria } & \multicolumn{2}{|c|}{ Gram Positive Bacteria } & \multicolumn{3}{|c|}{ FUNGAL SPECIES } \\
\hline & & & E. coli & P. aeruginosa & S. aureus & S. pyogenes & C.albicans & A. niger & A. clavatus \\
\hline 1. & T1GE & $\mathrm{H}$ & 250 & $>1000$ & 250 & 500 & 125 & 500 & $>1000$ \\
\hline 2. & T2GE & $2-\mathrm{CH}_{3}$ & 125 & 500 & 250 & 500 & 500 & 125 & 500 \\
\hline 3. & T3GE & $4-\mathrm{CH}_{3}$ & 500 & 250 & 500 & 250 & 1000 & 500 & 500 \\
\hline 4. & T4GE & $2-\mathrm{OCH}_{3}$ & 250 & 62.5 & 500 & 1000 & $>1000$ & 250 & 250 \\
\hline 5. & T5GE & $4-\mathrm{OCH}_{3}$ & 62.5 & 125 & 250 & 62.5 & 1000 & 250 & 125 \\
\hline 6. & T6GE & $3-\mathrm{NO}_{2}$ & 250 & 500 & 250 & 500 & 125 & 500 & 500 \\
\hline 7. & T7GE & $3-\mathrm{Cl}$ & 62.5 & 500 & 250 & 500 & 500 & 500 & 1000 \\
\hline 8. & T8GE & $4-\mathrm{Cl}$ & 62.5 & 125 & 500 & 62.5 & 250 & 500 & 125 \\
\hline 9. & T9GE & $4-\mathrm{F}$ & 125 & 125 & 62.5 & 500 & 250 & 1000 & 500 \\
\hline 10. & T10GE & $4-\mathrm{Br}$ & 125 & 250 & 62.5 & 125 & 500 & 1000 & 500 \\
\hline 11. & T11GE & $2-\mathrm{NO}_{2}$ & 250 & 250 & 500 & 125 & 1000 & 500 & $>1000$ \\
\hline 12. & T12GE & $3-\mathrm{OCH}_{3}$ & 125 & 500 & 1000 & 500 & 125 & 500 & 500 \\
\hline 13. & T13GE & $3-\mathrm{CH}_{3}$ & 250 & 500 & 1000 & 250 & 125 & 500 & 500 \\
\hline 14. & T14GE & $4-\mathrm{NO}_{2}$ & 250 & 250 & 62.5 & 250 & 500 & 250 & 250 \\
\hline 15. & T15GE & Naphthyl & 500 & 125 & 1000 & 500 & 500 & 250 & 1000 \\
\hline 16. & \multicolumn{2}{|c|}{ Ampicillin } & 100 & 100 & 100 & 250 & $*$ & $*$ & $*$ \\
\hline 17. & \multicolumn{2}{|c|}{ Chloramphenicol } & 50 & 50 & 50 & 50 & $*$ & $*$ & $*$ \\
\hline 18. & \multicolumn{2}{|c|}{ Griseofulvin } & $*$ & $*$ & $*$ & $*$ & 500 & 100 & 100 \\
\hline
\end{tabular}

\section{Result and Discussion}

Compounds T5GE, T7GE and T8GE exhibited excellent activity and T2GE, T9GE, T10GE and T12GE compounds exhibited good activity against $E$. coli as compared to Ampicillin. Compounds T5GE, T8GE, T9GE and T15GE exhibited good activity at $100-125 \mu \mathrm{g} / \mathrm{mL}$ activity and T4GE exhibited excellent activity as $62.5 \mu \mathrm{g} / \mathrm{mL}$ against $P$. aeruginosa as compared to Ampicillin. Compounds T9GE, T10GE and T14GE showed excellent activity at $62.5 \mu \mathrm{g} / \mathrm{mL}$ against $S$. aureus as compared to Ampicillin ( $\mathrm{MIC}=250$ $\mu \mathrm{g} / \mathrm{mL}$ ). Compounds T10GE, T11GE exhibited good activity at $100-125 \mu \mathrm{g} / \mathrm{mL}$ and compound $\mathrm{T} 5 \mathrm{GE}$ and T8GE showed excellent activity at $62.5 \mu \mathrm{g} / \mathrm{mL}$ against $S$. pyogenes as compared to Ampicillin (MIC $=100$ $\mu \mathrm{g} / \mathrm{mL}$ ).

Most of the compounds showed very good antifungal activity against Candida albicans, their MIC values were in the range between $(100-500 \mu \mathrm{g} / \mathrm{mL})$. As far as the anti-fungal activity are concerned for substituted thiourea derivatives of s-triazine compounds T1GE, T6GE, T12GE and T13GE showed excellent activity at $125 \mu \mathrm{g} / \mathrm{mL}$ and compounds T8GE and T9GE showed average activity at $250 \mu \mathrm{g} / \mathrm{mL}$ against $C$. albicans as compared to Griseofulvin ( $\mathrm{MIC}=500$ $\mu \mathrm{g} / \mathrm{mL}$ ). Whereas T2GE, T4GE, T5GE and T14GE compounds showed good activity against Aspergillus Clavatus as compared to Griseofulvin $(\mathrm{MIC}=100$ $\mu \mathrm{g} / \mathrm{mL})$.

\section{Conclusion}

In this article we have report a series of 4nitrobenzohydrazide and 6-(4-methoxyphenyl)-4phenyl-6H-1, 3-thiazin-2-amine and substituted thiourea linked s-triazine showing better activity. T5GE showed better antifungal activity compared to standard. All the synthesized compounds have been established by elemental analysis, IR, ${ }^{1} \mathrm{H}$ NMR and mass spectral data. So, there is a future in doing more work on the synthesized compounds as some of them showed good activity against standard drugs.

\section{Acknowledgement}

The authors thankful to Principal Dr. A. S. Patel, Navyug Science College, Surat for providing necessary research facility, SAIF Chandigarh for NMR data and Central Kashiba laboratory for providing antimicrobial activity. 


\section{References}

1. Moustafa M A, Gineinah M M, Nasr M N, Arch Pharma, (2004) 337,427-433.

2. Demain A L, Sanchez S, J Antibiot, (2009) 62,5-16.

3. Krchnak V, Holladay M W, Solid phase heterocyclic chemistry Chem Rev, (2002) 102,61-92.

4. Da Silva C M, Da Silva D L, Modolo L V, Alves R V, De Resende M A, Martins C V B, De Fatima A, J Adv Res, (2011) 2,1-8.

5. Mohini Y, Prasad R B N, Karuna M S L, Med Chem Res, (2013) 22,4360-6.

6. Shi L, Tan S H, Li H Q, Song Y C, Zhu H L, Tan R X, Eur J Med Chem, (2007) 2,558-64

7. Cheng L S, Tang J J, Luo H, Jin X J, Dai F, Yang Y, Qian Y P, Bioorg Med Chem Lett, (2010) 20, 2417-20.

8. Rollas S, Gulerman N, Erdeniz H, J Med Chem, (2002) 57, 171-4.

9. Bayrak H, Demirbas A, Demirbas N, Karaoglu S A, Eur J Med Chem, (2009) 44, 4362-6.

10. Kamble V U, Patil A S, Badami S P, J Incl Pheno Macro Chem, (2010) 68(3), 347-58.

11. Kaymakcioglu B, Elcin Oruc-Emre E, Unsalan S, Tabanca N, Khan S I, Earl D, Iscan G, Demirci F, Rollas S, Med Chem Res, (2012) 21, 3499-508.

12. Loncle C, Brunel J M, Vidal N, Dherbomez M, Letourneux Y, Eur J Med Chem, (2004) 39,1067-71.

13. Yamashita H, Ohno K, Amada Y, Hattori H, Funatsu Y O, Toya T, J Pharm Exp Ther, (2004) 308,127-33.

14. Rathod S P, Charjan A P and Rajput P R, Ras J Chem, (2010) 3,363-7.

15. Keerthi Kumar B, J Pharm Rese, (2011) 4,274-5.

16. Srikanth Jupudi et al Inter J Rese Pharm Chem, (2013) 3, 213-20.

17. Kalirajan R et al, J Chem Tech Rese, (2009) 1, 27-34.

18. Wang W, Zhao B, Chao X and Wenpeng W, Inter J Org Chem, (2012) 2, 117-20.

19. Meric A, Zerrin N and Ibrahim H, Med Chem Rese, (2014) 17, 30-41.

20. Beauchamp B, Hilpert and Wang, World Intellectual Property Organization, (2011) 165.

21. Levy S B, Marshall B, Nat Med, (2004) 10, S122-9.

22. Patel R V, Kumari P, Rajani D P, Pannecouque C, DeClercq E, Chikhalia K H, Med Chem, (2012) 4, 1053 65.

23. Mishra A R, Singh S, J Agric Food Chem, (2000) 48, 5465-8.

24. Patel D H, Chikhalia K H, Shah N K, Patel D P, Kaswala P B, Buha V M, J Enzyme Inhib Med Chem, (2010) 25, 121-5.

25. Kumar G J, Bomma S S, Srihari E, Shrivastava S, Naidu V G M, Srinivas K, Rao V J, Med Chem Res, (2013) 22, 5973-81.

26. Liu B, Lee Y, Zou J, Petrassi H M, Joseph R W, Chao W, Michelotti E L, Bukhtiyarova M, Springman E B, Dorsey B D, Bioorg Med Chem Lett, (2010) 20, 6592-6.

27. Dianzani C, Collino M, Gallicchio M, Fantozzi R, Samaritani S, Signore G, Menicagli R, J Pharm Pharmacol, (2006) 58,219-26.

28. Avupati V R, Yejella R P, Parala V R, Killari K N, Papasani V M R, Bioorg Med Chem Lett, (2013) 23, 5968-5970.

29. Bhat H R, Singh U P, Gahtori P, Ghosh S K, Gogoi K, Prakashe A, Singh R S, New J Chem, (2013) 37, 265462.

30. Malik G M, Patel T V, Journal of Asian Scientific Research, (2017) 7(6), 214-23. 